Supporting Information for

\title{
Synergistically Boosting Thermoelectric Performance of PEDOT:PSS/SWCNT Composites via Ion Exchange Effect and Promoting SWCNT Dispersion by Ionic Liquid
}

Wenjiang Deng, ${ }^{\dagger}$ Liang Deng, ${ }^{\dagger, *}$ Zhipeng Li, ${ }^{\dagger}$ Yichuan Zhang, ${ }^{* \dagger}$ and Guangming Chen $^{*}{ }^{\dagger}$

$\uparrow$ College of Materials Science and Engineering, Shenzhen University, Shenzhen 518055, P. R. China

+ College of Physics and Optoelectronic Engineering, Shenzhen University, Shenzhen 518060, China

* Corresponding authors.

E-mail address: chengm@szu.edu.cn (G. Chen), yichuan.zhang@szu.edu.cn (Y.Zhang) 


\section{Contents}

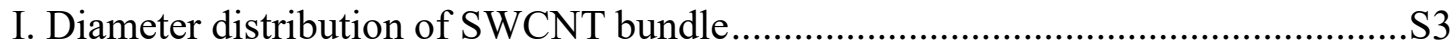

II. Optical photos of flexible PEDOT:PSS/SWCNT-IL50 film .................................S4

III. Water contact angles on different sample surfaces .............................................S5

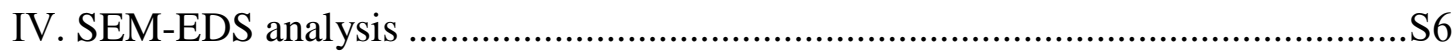

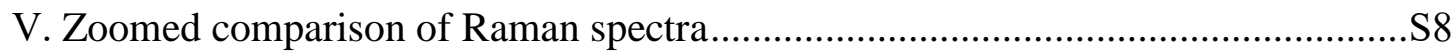

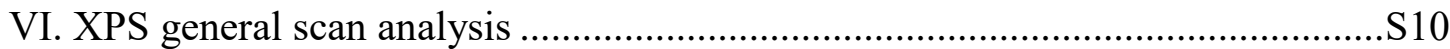




\section{The diameter distribution of SWCNT bundle}

Table S1. Parameters of the diameter distribution of SWCNT bundles fitted by a Gaussian distribution with $\mathrm{y}=y_{0}+A e^{-\left(x-x_{c}\right)^{2} / 2 \omega^{2}}$, where $x$ is the diameter of SWCNT bundles, $x_{c}$ is their mean value, $\omega$ is the standard deviation.

\begin{tabular}{ccccc}
\hline IL Content (wt $\%)$ & SWCNT Content $(\mathrm{wt} \%)$ & $x_{\mathrm{c}}(\mathrm{nm})$ & $\omega(\mathrm{nm})$ & $R^{2}$ \\
\hline \multirow{3}{*}{0} & 30 & 40.2 & 1.4 & 0.92 \\
& 60 & 41.9 & 2.6 & 0.92 \\
& 90 & 53.5 & 2.9 & 0.86 \\
\multirow{2}{*}{50} & 30 & 36.8 & 1.1 & 0.97 \\
& 60 & 38.1 & 0.8 & 0.97 \\
& 90 & 43.1 & 1.2 & 0.98 \\
\hline
\end{tabular}




\section{Optical photos of flexible PEDOT:PSS/SWCNT-IL50 film}

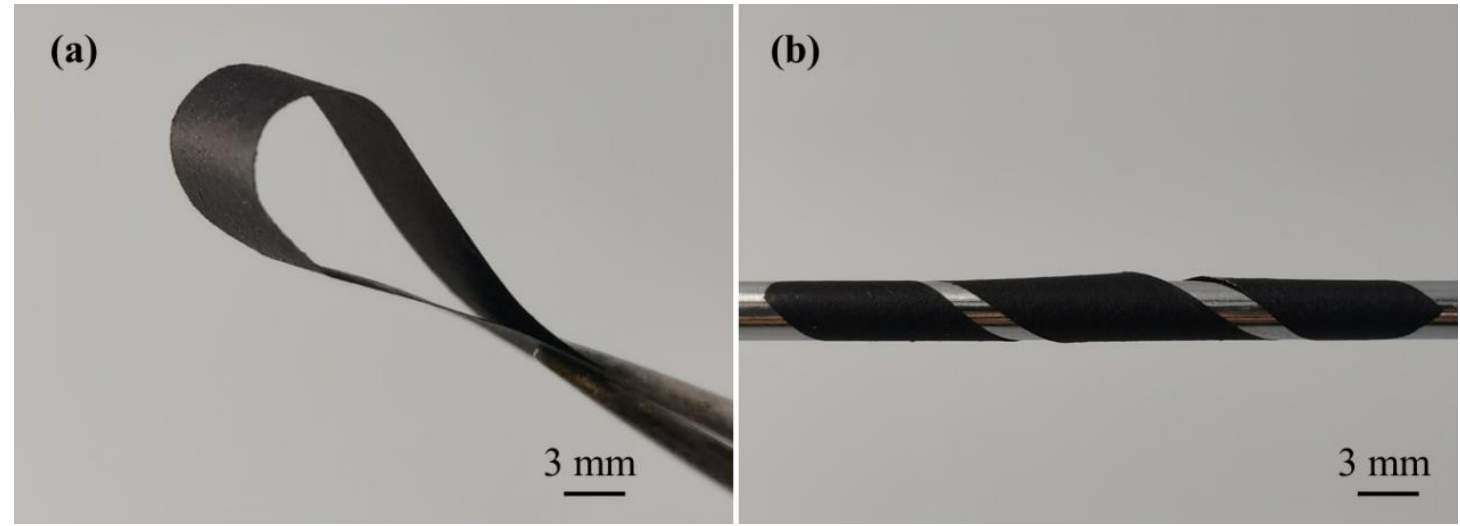

Figure S1. (a) Optical photo of flexible film of PEDOT:PSS/SWCNT-IL50 composite with $90 \mathrm{wt} \%$ SWCNT. (b) The film wrapping on a rod with radius of $1.5 \mathrm{~mm}$. Both photos confirm the high flexibility of the composite films. 
III. The water contact angles on different sample surfaces

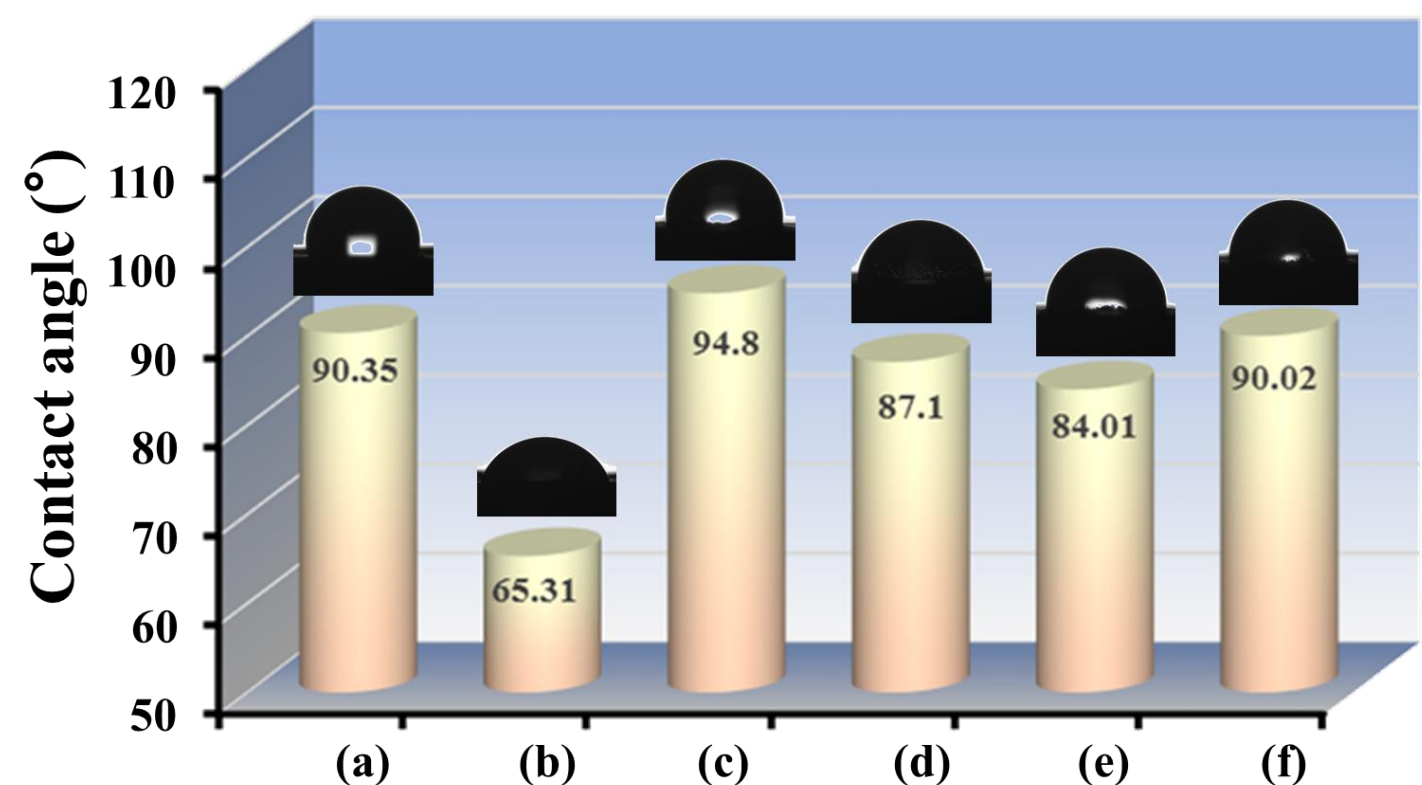

Figure S2. The water contact angles on the surfaces of (a) the pristine PEDOT:PSS film, (b) PEDOT:PSS film with $50 \mathrm{wt} \%$ IL, (c) IL-free PEDOT:PSS/SWCNT film, (d) PEDOT:PSS/SWCNT-IL25, (e) PEDOT:PSS/SWCNT-IL50 and (f) PEDOT:PSS/SWCNT-IL75. The labels of (a f) correspond to Figure 4 in the main text. 


\section{SEM-EDS analysis}
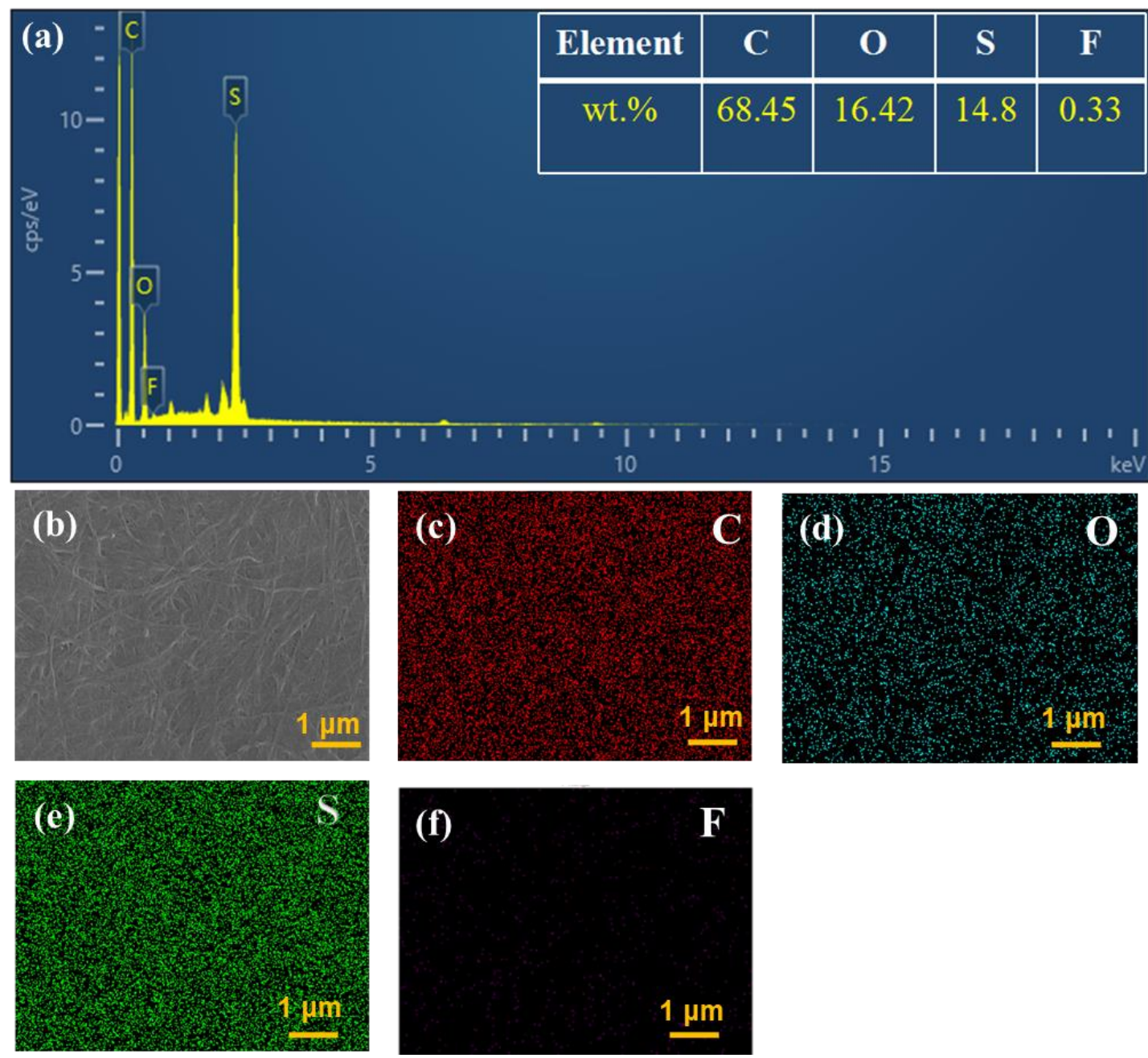

Figure S3. SEM-EDS analysis of the PEDOT:PSS/SWCNT-IL50 composite with 30 wt\% SWCNT. (a) EDS spectrum (the inset shows the element content), (b) SEM-EDS element mapping images and the corresponding EDS elemental mappings of (c) $\mathrm{C}$ element, (d) O element, (e) S element and (f) F element. 

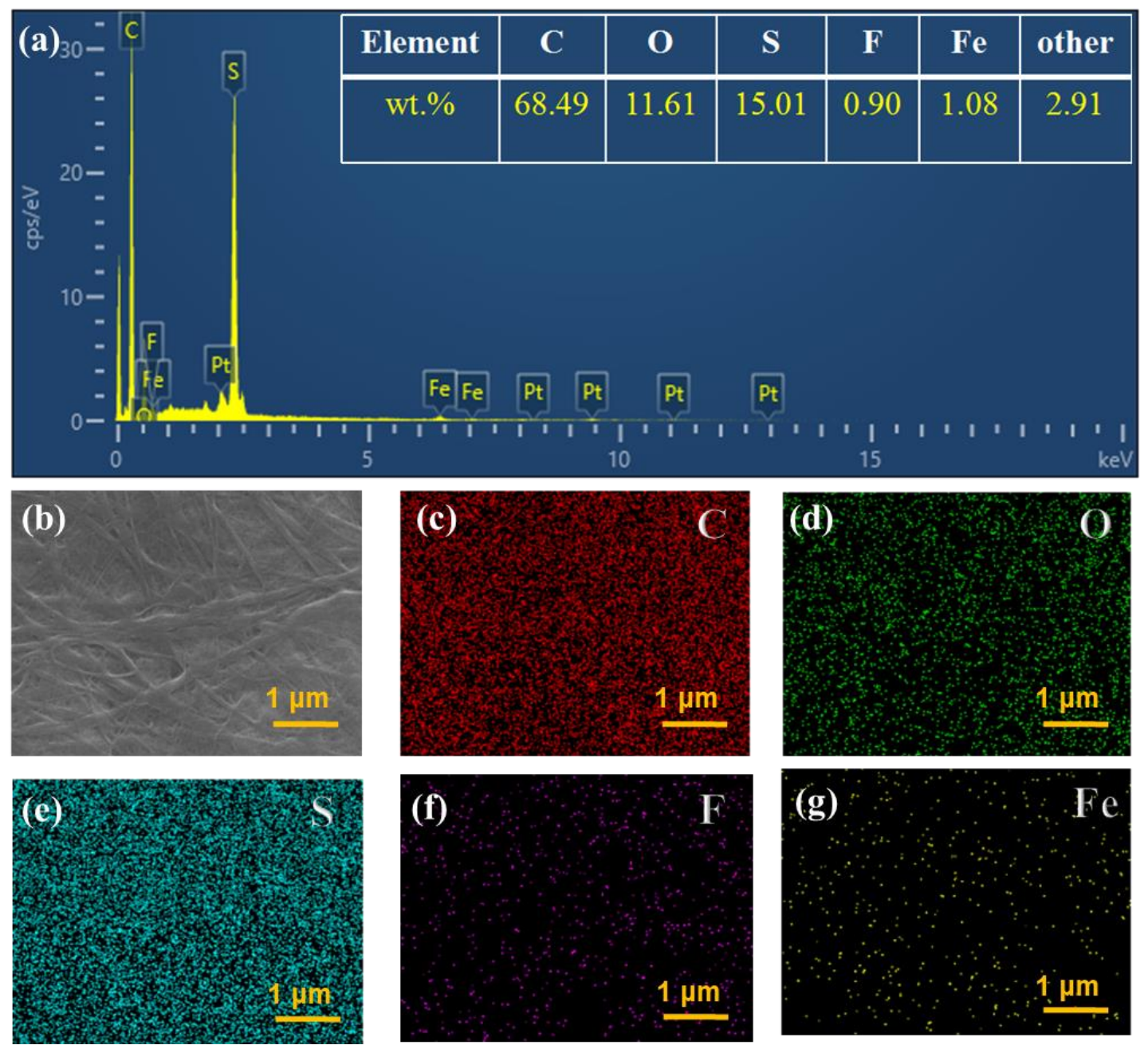

Figure S4. SEM-EDS analysis of the PEDOT:PSS/SWCNT-IL75 composite with 30

wt\% SWCNT. (a) EDS spectrum (the inset shows the element content), (b) SEM-EDS element mapping images and the corresponding EDS elemental mappings of (c) C element, (d) O element, (e) S element, (f) F element and (g) Fe element. 


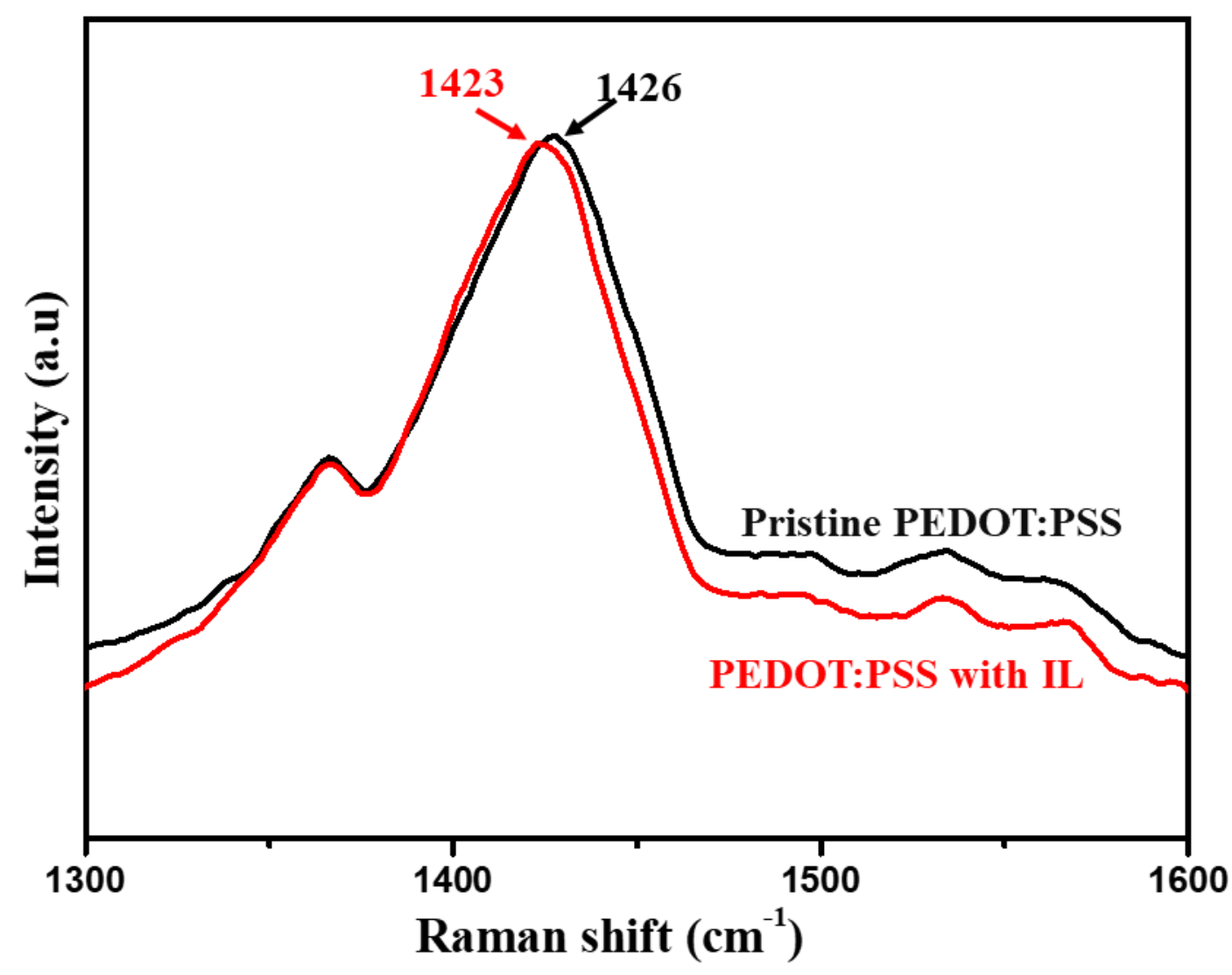

Figure S5. The zoomed comparison of Raman spectra for the pristine PEDOT:PSS film and the PEDOT:PSS film with IL. 


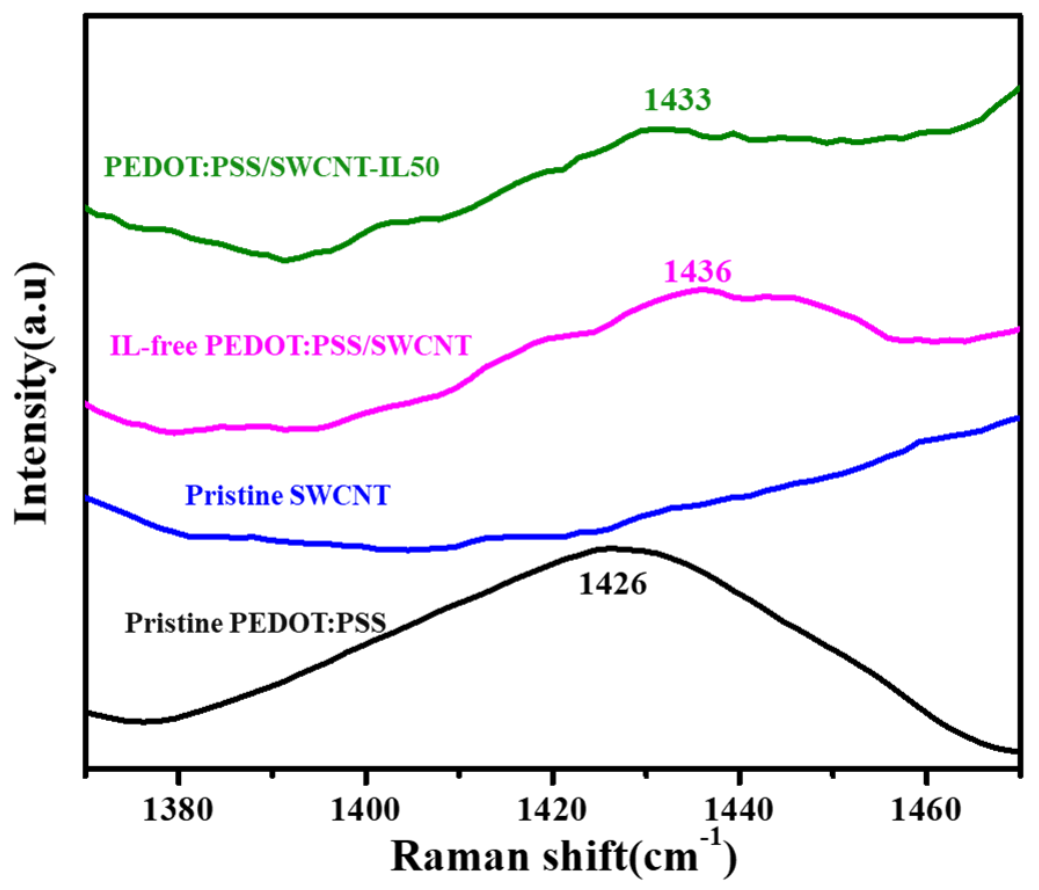

Figure S6. The zoomed comparison of Raman spectra for the pristine PEDOT:PSS , the pristine SWCNT, the IL-free PEDOT:PSS/SWCNT composite and the PEDOT:PSS/SWCNT-IL50 composite. The SWCNT contents are $30 \mathrm{wt} \%$ for both composite films. 


\section{XPS general scan analysis}

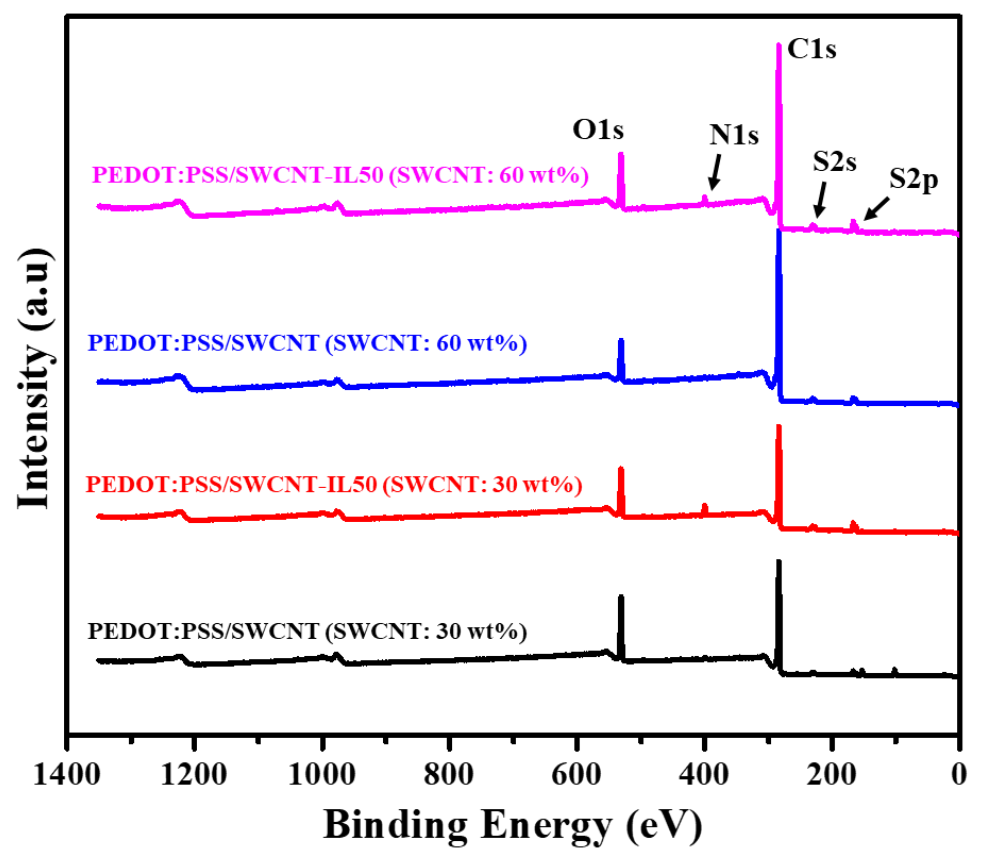

Figure S7. XPS general scan for the PEDOT:PSS/SWCNT composite (SWCNT: 30 wt\%), the PEDOT:PSS/SWCNT-IL50 composite (SWCNT: $30 \mathrm{wt} \%$ ), the PEDOT:PSS/SWCNT composite (SWCNT: $60 \mathrm{wt} \%$ ) and the PEDOT:PSS/SWCNTIL50 composite (SWCNT: $60 \mathrm{wt} \%$ ). 